\title{
Risk management in the treatment of type 2 diabetes with pioglitazone
}

This article was published in the following Dove Press journal:

Diabetes, Metabolic Syndrome and Obesity:Targets and Therapy 30 June 2009

Number of times this article has been viewed

\author{
Giuseppe Derosa \\ Sibilla AT Salvadeo \\ Department of Internal Medicine \\ and Therapeutics, University of Pavia, \\ Pavia, Italy
}

Introduction: Type 2 diabetes mellitus is one of the most important cardiovascular risk factors. Insulin-resistance represents the common mechanism that leads to type 2 diabetes in obese subjects. Pioglitazone is an insulin-sensitizing agent available for treatment of type 2 diabetes. Large clinical trials have demonstrated the effectiveness of pioglitazone in achieving metabolic control and reducing cardiovascular morbidity and mortality.

Aim: The purpose of this article is to review the effectiveness and tolerability of pioglitazone in the prevention and management of atherosclerosis in patients with type 2 diabetes.

Evidence review: We reviewed the main monotherapy and comparative studies of pioglitazone, and particularly the recent evidence in the field of atherosclerosis and cardiovascular prevention.

Place in therapy: The current evidence shows that pioglitazone is an effective option in the treatment of type 2 diabetes. More studies are needed to establish a role for pioglitazone in atherosclerosis prevention beyond glycemic control.

Keywords: pioglitazone, diabetes mellitus, glycated hemoglobin, cardiovascular prevention

\section{Introduction}

Around 246 million people are affected by type 2 diabetes, and this number is expected to be around 380 million by $2025 .{ }^{1}$ The associated annual mortality rate has been estimated to be around $5.5 \%$ of all patients. ${ }^{2}$ During the last decade, most of the commonly used drugs for diabetes have been shown to provide benefits on the cardiovascular (CV) system. Experimental evidence showed that metformin improves vascular function and procoagulant state by reducing circulating levels of plasminogen activator inhibitor- $1 . .^{3,4}$

Data from UKPDS in 1998 suggested that metformin could be responsible of a significant reduction of diabetes-related death and acute myocardial infarction (AMI) in obese patients initially randomized to monotherapy. ${ }^{5}$ The mechanism of action and the real contribution of metformin in reducing CV mortality was not definitively assessed in this study. An observational study has recently showed that treatment with metformin is associated with a lower CVD-related mortality rate compared to sulfonylurea monotherapy.

Although microvascular complications can lead to significant morbidity and mortality, the greatest cause of death in patients with type 2 diabetes is CV disease.

Data obtained over the past decade have demonstrated that the risk of microvascular complications of type 1 and type 2 diabetes can be reduced by intensive glycemic control. ${ }^{5,6}$ According to results of two large clinical trials, the Diabetes control and
Correspondence: Giuseppe Derosa Department of Internal Medicine and Therapeutics, University of Pavia, Pavia, Italy Email giuseppe.derosa@unipv.it 
Complications Trial (DCCT) and the UK Prospective Diabetes Study (UKPDS), the American Diabetes Association (ADA) recommends a glycosylated hemoglobin $\left(\mathrm{HbA}_{1 \mathrm{c}}\right.$ ) value $<7 \%$ for most subjects with diabetes mellitus, with more or less strict control in particular groups of patients and conditions (ie, pregnancy).

The relationships between strict glycemic control, represented by adequate values of glycosylated hemoglobin $\mathrm{A}_{1 \mathrm{c}}\left(\mathrm{HbA}_{\mathrm{lc}}\right)$, and $\mathrm{CV}$ disease are still less clearly defined, despite results of DCCT and UKPDS. Recently published results of tree large clinical trials have further enriched the acknowledgement about a direct effect of tight glycemic control and reduction of $\mathrm{CV}$ disease in patients with type 2 diabetes. Two of these studies, Action in Diabetes and Vascular Disease Preterax and Diamicron modified Release Controlled Evaluation $(\text { ADVANCE) })^{7}$ and Veterans Affairs Diabetes Trial (VADT) ${ }^{8}$ have shown that $\mathrm{CV}$ outcomes are not significantly reduced by strict glycemic control. On the other hand, a third trial, the action to Control Cardiovascular risk in Diabetes (ACCORD), ${ }^{9}$ has been stopped earlier than expected due to a significant increase in mortality in participants randomized to very intensive glycemic control $\left(\mathrm{HbA}_{1 \mathrm{c}}<6 \%\right)$. According to these findings, the ADA revised recommendations for glycemic targets in patients with diabetes mellitus.

A well thought out analysis of the studies published in 2008 (ACCORD, STENO 2 post-trial, ADVANCE, VADT, UKPDS post-trial, Epidemiology of Diabetes Interventions and Complications [EDIC]) highlighted the existence of glycemic memory, the non-existence of blood pressure memory, the need to control all CV risk factors and to treat diabetes mellitus early while avoiding hypoglycemic incidents. The glycemic target based on $\mathrm{HbA}_{1 \mathrm{c}}$ must take into account the patient's age and the duration of diabetes, as well as cardiovascular risk factors and previous glycemic control. All in all, the intensive treatment of type 2 diabetes must begin early; it must not be too rapid and must avoid hypoglycemic incidents and be combined with a strict control of other $\mathrm{CV}$ risk factors. ${ }^{10}$

In addition to lowering blood glucose levels and ameliorating lipids concentration, thiazolidinediones (TZDs) have anti-inflammatory and antithrombotic effects. In fact both pioglitazone and rosiglitazone have been shown to improve endothelial dysfunction. ${ }^{11}$

Recently, two large clinical trials, PROactive and PERISCOPE, have provided data suggesting a role of pioglitazone in preventing $\mathrm{CV}$ events in patients with type 2 diabetes mellitus. Whether these effects translate into beneficial CV outcomes is still controversial and CV side effects, such as heart failure (HF), have been reported in clinical trials of TZDs. ${ }^{12,13}$

Rosiglitazone trials with CV outcomes are ongoing. Data from the RECORD (Rosiglitazone Evaluated for Cardiac Outcome and Regulation of Glycemia in Diabetes) study have been recently published in an interim analysis and show no statistically significant difference between rosiglitazone and placebo in preventing AMI, death from CV causes or death from any other causes..$^{14}$

In this manuscript we highlight the efficacy and safety of pioglitazone, a TZD, in improving a number of well established and recently recognized CV risk factors which need to be addressed in diabetes mellitus management.

\section{Efficacy studies with pioglitazone}

A large number of data are available on the efficacy of pioglitazone in glycemic control. In a study involving 408 patients with type 2 diabetes mellitus, who underwent discontinuation of previous therapy, pioglitazone significantly reduced $\mathrm{HbA}_{1 \mathrm{c}}$ and fasting plasma insulin (FPI) after 3 months, while reduction in fasting plasma glucose (FPG) was reached after a longer period. ${ }^{15}$ A post-hoc analysis of these results showed a significant increase in insulinsensitivity, expressed by Homeostasis Model Assessment index (HOMA index) ( $<0.05$ with $15 \mathrm{mg} /$ day and $\mathrm{p}<0.001$ with increased doses from 30 to $45 \mathrm{mg}$ die). ${ }^{16}$

In studies with low doses of pioglitazone (15 or $30 \mathrm{mg} /$ day), the effects on $\mathrm{HbA}_{1 \mathrm{c}}$ and FPG were comparable with the two dosages of administration. Moreover, both dosages required a 4-week period before their effects could be demonstrated. Even when pioglitazone, at doses ranging from 30 to $45 \mathrm{mg}$ /day, was administered to patients with normal to moderate levels of $\mathrm{HbA}_{1 \mathrm{c}}$, HOMA index and FPG were significantly improved ( $\mathrm{p}<0.001$, for both parameters). In two large studies, pioglitazone induced a significant improvement of high-density lipoprotein cholesterol (HDL-C) $(p<0.001)$, without significant differences between different dosages, while high doses, $45 \mathrm{mg} /$ day, were related to significant reduction of triglycerides $(\mathrm{Tg})$ and apolipoprotein B (Apo B) $(\mathrm{p}<0.01$ and $\mathrm{p}<0.05)$. No low-density

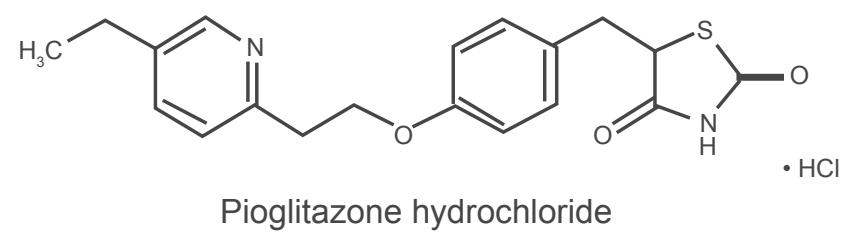

Figure I Pioglitazone chemical structure. 
lipoprotein (LDL-C) reduction was observed in either study, even though high-density lipoproteins/low-density lipoproteins (HDL/LDL) ratio was improved in one study. ${ }^{17,18}$

Pioglitazone, administered in monotherapy at dosage of $30 \mathrm{mg} /$ day, has been investigated in a large study including 197 subjects with type 2 diabetes mellitus. Pioglitazone significantly reduced FPG, $\mathrm{HbA}_{1 \mathrm{c}}$, C-peptide and FPI $(\mathrm{p}<0.05$, for all comparisons). In conclusion, pioglitazone seemed to beneficially affect both glycemic control parameters and lipid profile in terms of reduction in $\mathrm{Tg}$ and increase in HDL-C, with small effects on LDL-C (Table 1). ${ }^{19}$

When pioglitazone is compared with metformin, it shows a similar effect in glycemic control. In one study, involving 205 patients with type 2 diabetes, both pioglitazone and metformin reduced $\mathrm{HbA}_{1 \mathrm{c}}(-1.3 \%$ and $-1.5 \%, \mathrm{p}<0.0001$, respectively), FPG $(-3.0$ and $-2.8 \mathrm{mmol} / \mathrm{L}, \mathrm{p}<0.001$, respectively) and FPI $(-17.0 \%, \mathrm{p}<0.01$ vs baseline; $-14.9 \%$ vs baseline, $\mathrm{p}<0.05$, respectively) after 24 weeks. At the end of the study, pioglitazone showed a greater effect on reduction of FPI than metformin ( $\mathrm{p}<0.05$ for comparison). ${ }^{20}$

In another study, pioglitazone reduced FPG $(-2.5$ vs $-2.2 \mathrm{mmol} / \mathrm{L}, \mathrm{p}<0.05$, respectively) and $\mathrm{Tg}(-0.61$ vs $-0.30 \mathrm{mmol} / \mathrm{L}, \mathrm{p}<0.001$, respectively) to a greater extent than metformin. Moreover, pioglitazone showed a major effect on HDL-C (0.16 vs $0.08 \mathrm{mmol} / \mathrm{L} ; \mathrm{p}<0.001)$, FFA $(0.16 \mathrm{vs}-0.08 \mathrm{mmol} / \mathrm{L} ; \mathrm{p}<0.001)$ and urinary albumin excretion rate (AER) $\left(-19.0 \% \mathrm{vs}-1.0 \%, \mathrm{p}<0.005\right.$, respectively). ${ }^{21}$

One of the main issues in type 2 diabetes management is preventing the progressive impairment of pancreatic $\beta$-cell function. Studies in vivo reveal that TZDs promote $\beta$-cell survival and regranulation as well as maintenance of $\beta$-cell mass and reduction of amyloid deposition. Recent data are available which support the notion that TZDs have

Table I Pioglitazone effects on lipoproteins

\begin{tabular}{ll}
\hline Site & Effects \\
\hline Blood & $\downarrow$ Tg \\
& $\downarrow$ FFA \\
& $\downarrow$ Lipid perox \\
& $\downarrow$ TNF- $\alpha$ \\
& $\downarrow$ Resistin \\
& $\downarrow$ sdLDL \\
& $\uparrow$ HDL-C \\
& $\uparrow$ LDL I, HDL 2 \\
& $\uparrow$ Transport proteins of FFA \\
& $\downarrow$ ApoCIII \\
Adipose tissue & $\uparrow$ ApoAl \\
\hline
\end{tabular}

Abbreviations: $\mathrm{Tg}$, triglycerides; ApoCIII, apolipoprotein CIII; FFA, free fatty acids; ApoAl, apolipoprotein Al; sdLDL, small-dense LDL; HDL-C, high-density lipoprotein cholesterol; LDL, low-density lipoprotein. beneficial effects on $\beta$-cell function. These clinical studies demonstrated that TZDs are able to prevent or delay the development of type 2 diabetes mellitus in a high-risk population through restoration of the first-phase insulin response and improvement of secretory responses to oscillations in plasma glucose levels. Many of these effects appear to be independent of improvement in insulin sensitivity. Emerging evidence suggests that TZDs offer specific benefits for preventing or delaying the decline in $\beta$-cell function and, thereby, a substrate for early intervention efforts aimed at lowering the burden type 2 diabetes mellitus. ${ }^{22}$

Compared with sulfonylureas, pioglitazone produces a greater reduction of $\mathrm{HbA}_{1 \mathrm{c}}$, and improves insulin sensitivity. Compared with glibenclamide, pioglitazone increases insulin sensitivity (HOMA index $13.0 \%$ vs $17.0 \%$, p $<0.001$, respectively) and $\beta$-cell function after 36 weeks. Results of comparator studies suggest that even if pioglitazone effects on $\mathrm{HbA}_{1 \mathrm{c}}$ require a longer period to be evident, pioglitazone allows a more sustained glycemic control and improvement in insulin sensitivity than glibenclamide. ${ }^{23}$

Results from clinical studies clearly show a substantial superiority of pioglitazone in ameliorating lipids in diabetes patients, compared with other molecules. Pioglitazone significantly increases HDL-C while both glimepiride and glibenclamide do not affect this parameter. Compared with glibenclamide, pioglitazone significantly reduces $\mathrm{Tg}$, but, compared with glimepiride, similar and non-significant effects on Tg have been observed for both drugs. Both pioglitazone and glibenclamide induce a moderate increase in LDL-C. ${ }^{24,25}$

For lipoprotein subfractions, pioglitazone increases the larger subfractions of LDL-C and HDL-C compared with metfomin and glibenclamide, and reduces small-dense LDL-C subfractions (sdLDL), which are ameliorated also by metformin. ${ }^{16}$

Some data suggest a superior effect of pioglitazone in association with metformin, compared with treatment with rosiglitazone and metformin. In a recent study, significant total cholesterol (TC), LDL-C, HDL-C, Tg, apolipoprotein AI (Apo AI), and Apo B were ameliorated in the pioglitazone group, but not in the rosiglitazone group, after 12 months. These variations were significant between groups $(\mathrm{p}<0.05){ }^{26}$

In our experience, an association of pioglitazone or rosiglitazone and glimepiride shows a greater effect of pioglitazone on lipid profile in patients with type 2 diabetes no longer responding to glimepiride. In one study, pioglitazone in association with glimepiride significantly 
improved TC (-11.0\%), LDL-C (-12.0\%), HDL-C (15.0\%) and Apo B $(-10.6 \%)$ ( $\mathrm{p}<0.05$ for all parameters), compared with add-on therapy with rosiglitazone, which worsened the same parameters (Table 2). ${ }^{27}$

In a meta-analysis by Iki-Yarvinien, both pioglitazone and rosiglitazone increased HDL-C (10\%) to a similar extent, but pioglitazone has shown a better effect on $\mathrm{Tg}(20 \%){ }^{28}$

Accumulating evidence clearly shows that TZDs affect $\mathrm{CV}$ risk factors. There is no clear statement about the real effects of TZD on the single recognized parameter that increases CV risk, while much evidence is available on the so-called ancillary effects of TZDs.

Pioglitazone seems to be as efficacious as rosiglitazone in reducing systolic blood pressure (SBP) and diastolic blood pressure (DBP). In a study on non-dipper subjects with type 2 diabetes mellitus, patients treated with metformin and pioglitazone underwent a significant improvement of ambulatory BP monitoring after 8 weeks, not observed with metformin monotherapy and independent of changes in glycemic control. ${ }^{29}$

In another similar study, both pioglitazone and rosiglitazone in association with glimepiride and metformin significantly reduced SBP and DBP in 87 subjects with type 2 diabetes $(\mathrm{p}<0.05)$, independently of improvement in metabolic parameters. ${ }^{30}$

In a recent 12-month study, the combination of TZDs and metformin was found to be associated with a slight, but significant, improvement in the long-term BP control $(\mathrm{p}<0.05)$, related to a similar reduction in insulin resistance. In association with metformin, both pioglitazone and rosiglitazone add-on therapy appear to significantly improve BP control. ${ }^{31}$

Available data suggest that pioglitazone is effective in reducing AER, both in monotherapy and in combination with metformin or sulfonylureas, as demonstrated in the large QUARTET study. Compared with metformin plus sulfonylureas, addition of pioglitazone to a sulfonylurea resulted in reduction of the AER, a small but significant rise in LDL-C, and significantly greater improvements of Tg and HDL-C levels. ${ }^{32}$

In another study, a combination of pioglitazone and metformin has been compared with a combination of gliclazide and metformin. Pioglitazone add-on therapy has been shown to be more effective than gliclazide in reducing AER. ${ }^{33}$

Emerging data suggest pioglitazone effectiveness in prevention and treatment of early organ damage.

Results from a comparative study have suggested that, despite similar improvements in metabolic control $\left(\mathrm{HbA}_{1 \mathrm{c}}\right)$, a 24-week treatment with pioglitazone significantly improved intima-media thickness $(-0.033 \pm 0.052 \mathrm{~mm}$; $\mathrm{p}<0.0001)$, pro-insulin intact concentration $(-5.92 \pm 10.04 \mathrm{pmol} / \mathrm{L}$; $\mathrm{p}<0.0001)$, adiponectin (ADN) $(10.9 \pm 6.3 \mu \mathrm{g} / \mathrm{mL}$; $\mathrm{p}<0.0001)$ and HOMA index $(-2.21 \pm 3.40 ; \mathrm{p}<0.0001)$, compared with glimepiride. Reduction in intima-media thickness has been correlated with improved insulin sensitivity $(\mathrm{r}=0.29 ; \mathrm{p}=0.0003)$, and pro-insulin intact levels $(\mathrm{r}=0.22$; $\mathrm{p}=0.006$ ), while an inverse correlation has been found with ADN levels $(\mathrm{r}=-0.37 ; \mathrm{p}<0.0001) .{ }^{34}$ Moreover, other data suggest that pioglitazone slows progression of intima-media thickness. $^{35}$

Other studies are available about the effects of pioglitazone treatment on endothelium-dependent vasodilation. In a small study, pioglitazone did not decrease FPG and $\mathrm{HbA}_{1 \mathrm{c}}$ in non-diabetes subjects but significantly improved endotheliumdependent vasodilation measured by flow-mediated dilation $(5.0 \% \pm 2.2 \%$ to $6.3 \% \pm 2.4 \%, \mathrm{p}<0.05) .{ }^{36}$

In a small study involving patients with type 2 diabetes mellitus or impaired glucose tolerance (IGT) who were previously treated with percutaneous coronary intervention, pioglitazone significantly reduced coronary plaques volume after 6 months $\left(101.3 \pm 32.1\right.$ to $94.6 \pm 33.6 \mathrm{~mm}^{3},-7.2 \%$; $\mathrm{p}=0.0023)$ and plasma $\mathrm{Tg}(-14.9 \%)$, while HDL-C was

Table 2 Thiazolidinediones and lipid profile: experience of the authors

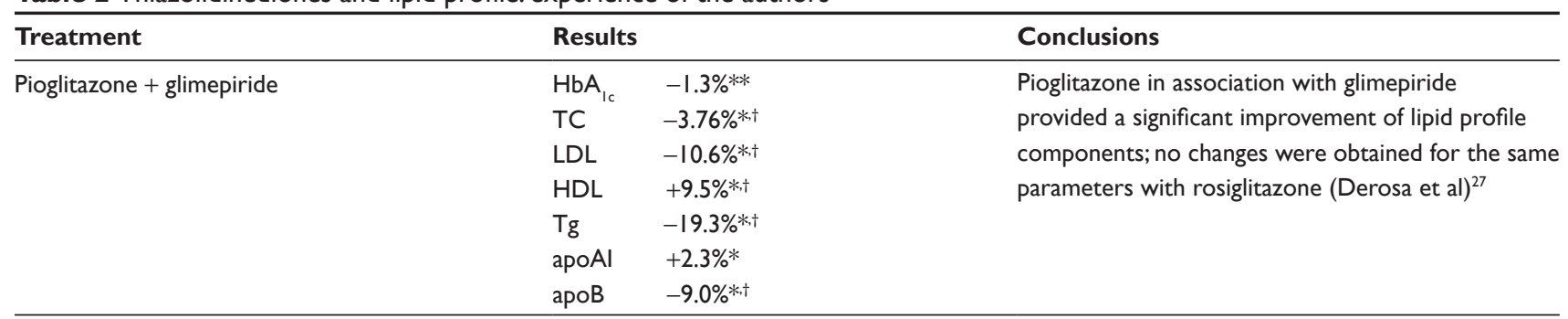

$*_{\mathrm{p}}<0.05$ vs baseline; ${ }^{*} \mathrm{p} p<0.01$ vs baseline; ${ }^{\dagger} \mathrm{p}<0.05$ vs the comparative treatment.

Abbreviations: $\mathrm{HbA}_{\mathrm{Ic}}$, glycosylated hemoglobin; TC, total cholesterol; LDL-C, low-density lipoprotein; HDL-C, high-density lipoprotein; Tg, triglycerides; apoAl, apolipoprotein Al; apoB, apolipoprotein B. 
substantially increased $(+20.0 \%)$, without any significant change in LDL-C. Furthermore, high sensitivity C-reactive protein (hs-CRP) level decreased. ${ }^{37}$

Significant effects of pioglitazone on non-conventional $\mathrm{CV}$ risk factors, such as lipoprotein (a) [Lp(a)] and homocystein (HCT) have been recently demonstrated. In our experience significant $\mathrm{Lp}(\mathrm{a})$ and $\mathrm{HCT}$ improvement has been obtained after a 12-month treatment with pioglitazone compared with baseline values, and Lp(a) change was significant, compared with the rosiglitazone group. Significant HCT decrease was observed in the rosiglitazone group at the end of the study. However, long-term treatment with metformin plus pioglitazone significantly reduced Lp(a) plasma levels, whereas metformin combined with rosiglitazone did not (Table 3). ${ }^{38}$

Results of two large clinical trials, the PROactive study and the PERISCOPE study, recently published, have clearly highlighted the role of pioglitazone in prevention of $\mathrm{CV}$ events. ${ }^{12,13}$

The PRO-active (PROspective pioglitAzone Clinical Trial in macroVascular events) has showed that treatment with pioglitazone is effective in improving a series of $\mathrm{CV}$ risk factors, resulting in a general reduction of $\mathrm{CV}$ morbidity and mortality. Tolerability of pioglitazone at different dosages (from $15 \mathrm{mg}$ /day to $45 \mathrm{mg} /$ day) has been evaluated, too. All patients enrolled presented macrovascular disease such as recent ( $<6$ months) AMI, acute coronary syndrome, re-vascularization, stroke, or severe peripheral arterial disease. The composite primary end-point was the time required for the presentation of a second $\mathrm{CV}$ event after randomization: death from all causes, non-fatal myocardial infarction (including silent AMI), stroke, acute coronary syndrome, coronary or peripheral revascularization, and amputation. Secondary end-points were the time preceding the first all-cause death, AMI (excluding silent AMI) and stroke (main secondary end-point). The primary endpoint was reduced by $10 \%$ by pioglitazone ( 15 to $45 \mathrm{mg}$ ) (pioglitazone 514/2605 events vs placebo 572/2633, hazard ratio [HR] 0.90 95\% confidence interval [CI] 0.80-1.02; $\mathrm{p}=0.095$ ). When the first end-point is given only from clinical objectives, it was significant for pioglitazone by $16 \%$ for the pioglitazone group vs placebo $(p=0.027)$. Including re-vascularization events in the primary composite end-point actually reduces the significance of the results since revascularization depends on the different possibility of different centers to conduct this surgical therapy. In conclusion, PROactive showed a substantial constant reduction of AMI, stroke, acute coronary syndrome, and cardiac re-vascularization in patients treated with pioglitazone compared with placebo, and represents the first clinical trial that demonstrated a possible reduction of $\mathrm{CV}$ morbidity and mortality in patients with type 2 diabetes. ${ }^{12}$

PERISCOPE (Pioglitazone Effect on Regression of Intravascular Sonographic Coronary Obstruction Prospective Evaluation) is a randomized, double-blind, multi-center study, whose results have been recently published, which involved 543 subjects with coronary disease and type 2 diabetes mellitus. The main outcome was the ultrasonographic measure of percentage atheroma value (PAV) before and after 18 months' treatment with glimepiride (1-4 mg/day) or pioglitazone (15-45 mg/day). At the end of the study, the mean PAV had been reduced by $16 \%$ in the pioglitazone group and increased of $0.73 \%$ by the glimepiride group $(\mathrm{p}=0.002)$. Pioglitazone significantly reduced $\mathrm{HbA}_{1 \mathrm{c}}$, to a major extent,

Table 3 Thiazolidinediones and non-conventional cardiovascular risk factors: experience of the authors

\begin{tabular}{|c|c|c|c|}
\hline Treatment & Resul & & Conclusions \\
\hline Pioglitazone + metformin & $\begin{array}{l}\mathrm{HbA}_{\text {Ic }} \\
\text { TC } \\
\text { LDL } \\
\text { HDL } \\
\text { Tg } \\
\text { LP (a) } \\
\text { HCT }\end{array}$ & $\begin{array}{l}-1.0 \% * * \\
-6.4 \%^{*, t} \\
-5.2 \%^{*, \dagger} \\
+4.34 \%^{*, \dagger} \\
-19.2 \%^{*, \dagger} \\
-10.4 \%^{*, \dagger} \\
-17.3 \%^{*}\end{array}$ & $\begin{array}{l}\text { Pioglitazone in association with metformin } \\
\text { provided a significant improvement in Lp(a) } \\
\text { concentration (Derosa et al) }{ }^{38}\end{array}$ \\
\hline Pioglitazone + glimepiride & $\begin{array}{l}\mathrm{HbA}_{\mathrm{Ic}} \\
\mathrm{Tg} \\
\mathrm{PAl}-\mathrm{I} \\
\mathrm{t}-\mathrm{PA} \\
\mathrm{Fg}\end{array}$ & $\begin{array}{l}-1.1 \% * * \\
-19.0 \% *, \dagger \\
-18.0 \% * \\
-16.8 \% \\
-3.6 \%\end{array}$ & $\begin{array}{l}\text { Combination treatment produced a slight but } \\
\text { significant reduction of PAI-I concentration } \\
(\text { Derosa et al) })^{30}\end{array}$ \\
\hline
\end{tabular}

${ }^{*} \mathrm{p}<0.05$ vs baseline; ${ }^{* *} \mathrm{p}<0.01$ vs baseline; ${ }^{\dagger} \mathrm{p}<0.05$ vs the comparative treatment.

Abbreviations: $\mathrm{HbA}_{1 c}$, glycosylated hemoglobin; TC, total cholesterol; LDL-C, low-density lipoprotein; HDL-C, high-density lipoprotein; Tg, triglycerides; Lp(a), lipoprotein(a); $\mathrm{HCT}$, homocysteine; PAI-I, plasminogen activator inhibitor I; t-PA, tissue-plasminogen activator; Fg, fibrinogen. 
compared with glimepiride ( $-0.55 \%$ vs $0.36 \%$; respectively, $\mathrm{p}=0.03$ between groups), and increased HDL-C (+16\% vs $4.1 \%)$ and $\mathrm{Tg}$ median value $(-15.3 \%$ vs $0.6 \%)(\mathrm{p}<0.001$ for both parameters). PERISCOPE is the first study to demonstrate that pioglitazone can reduce coronary atheroma. As a great percentage (90\%) of patients involved in this study was treated with an optimal therapy for glycemic and dyslipidemia control, pioglitazone has been shown to be able to add benefit to the recommended therapy. The authors concluded that there are many explanations for the mechanisms that lead to such encouraging results. PERISCOPE investigators have reported adverse events, even if clinical outcomes were not pre-specified in the study. In particular, edema and bone fractures $(3.0 \%)$ were more frequent in patients treated with pioglitazone. Increases in body weight were observed in both groups, but differences were not significant. ${ }^{13}$

\section{Safety and tolerability}

Edema represents one of the most common and undesirable side effects of treatment with a TZD. Edema seems more common when TZDs are used in combination therapy. In the Matthew study, the incidence of edema in patients treated with pioglitazone and metformin was $6.3 \%$ and $2.2 \%$ in patients treated with gliclazide and metformin. ${ }^{33}$ Accumulating data support an increased risk of edema in patients treated with pioglitazone. In a study by Einhorn et al the incidence of peripheral edema increased by $5.9 \%$ in the group treated with pioglitazone and metformin compared with the group treated with metformin alone, even if the events in this study were described as mild to moderate. ${ }^{39}$ Fluid retention with TZDs may be considered a multi-factorial process. Excluding association with insulin, among combination treatments, the major incidence of edema has been reported when pioglitazone or rosiglitazone are associated with sulfonylureas. ${ }^{40}$

There are data supporting an increased risk of HF with pioglitazone. Both pioglitazone and rosiglitazone cause fluid retention and potentially promote congestive heart failure (CHF).

The mechanisms underlying this phenomenon have not been yet clearly described, even if some hypotheses have been proposed. Among these, the most studied are the increased expression of vascular endothelial growth factor, which simulates endothelial permeability, ${ }^{41}$ and the possible inhibition of A and B type natriuretic peptides, demonstrated in an in vitro model. ${ }^{42}$ In a recent study, echocardiographic parameters were not altered in patients treated for 3 months with pioglitazone $30 \mathrm{mg} /$ day who have undergone an increase in N-terminal pro-B-type natriuretic peptide (NT-proBNP).
In particular, no functional impairment has been observed in patients with basal high NT-proBNP. ${ }^{43}$

Analysis of recent data shows that treatment of type 2 diabetes mellitus with a TZD increases signs of CHF, but not the risk of $\mathrm{CV}$ and overall death, and type 2 diabetics with preexisting HF benefit from a reduction in CV end-points. ${ }^{44}$

Results on the effects of TZDs on bone in humans are contrasting and few. Data available suggest that treatment with TZDs, primarily rosiglitazone, contributes to bone loss. The effect appears to be most prominent in post-menopausal women. More studies are needed to better understand the effects of TZDs on bone and fracture rates. As with all TZDs, pioglitazone increases the risk of osteoporosis. The mechanisms by which pioglitazone reduces bone mass density have not been yet elucidated, even if recent data show that TZDs can influence differentiation of osteobasts. ${ }^{45}$ Few studies have evaluated differential effects of pioglitazone, rosiglitazone and troglitazone on bone loss. In a recently published study, hip body mass index (BMD) was measured by dual-energy $\mathrm{X}$-ray absorptiometry in patients with type 2 diabetes treated with troglitazone, pioglitazone and rosiglitazone. Each year of treatment with TZD was associated with greater bone loss of the whole body in women but not in men. No data were provided about a possible differential effect of each TZD on bone loss, although this possibility has been suggested in other studies with TZD. ${ }^{46,47}$

Recently, TZDs have been evaluated in studies of treatment of polycystic ovary syndrome (PCOS). In one study, pioglitazone treatment was followed by a significant decrease of hip and lumbar BMD and parameters of bone turnover in premenopausal women with PCOS, who are considered protected against bone loss by hyperinsulinemia. The mechanism of bone mineral loss in this and other studies has not been yet clarified and long-term intervention studies are required to elucidate the possible effects of pioglitazone on bone structure and turnover. ${ }^{48}$

No studies have directly evaluated differences in pioglitazone tolerability between human males and females. Recently the hypothesis that some adverse effects of TZDs could be related to specific interactions between these molecules and sex steroids has been proposed. Pioglitazone and rosiglitazone have been suggested to reduce testosterone biosynthesis and function. This hypothesis starts from the evidence of a possible link between low levels of testosterone and development of type 2 diabetes mellitus. An augmented incidence of side effects with pioglitazone and rosiglitazone may be further explained by a possible down-regulation of androgen biosynthesis. ${ }^{49}$ In a randomized controlled study, 
38 men with moderate to severe erectile dysfunction with poor response to sildenafil were pre-medicated with pioglitazone or placebo. Pioglitazone significantly improved major outcome measures, without affecting testosterone and dehydroepiandrosterone levels. Stronger conclusions about the interaction between pioglitazone and hormones would be obtained by investigating the effects of pioglitazone on sex steroids in larger randomized clinical trials. ${ }^{50}$

Aminotransferase increase represents another common problem that could be related to use of TZDs. In a 3year, randomized, double-blind safety study the incidence of alanine aminotransferase (ALT) elevation $\geq 3$ times the upper limit during treatment with pioglitazone and metformin was comparable to that with glibenclamide and metformin. In the same study, the incidence of ALT and gamma-glutamyl-transpeptidase $(\gamma-\mathrm{GT})>1.5$ the upper limit and/or baseline was significantly more frequent with glibenclamide than with pioglitazone in association with metformin. Aspartate aminotransferase (AST) elevation was more frequent with pioglitazone but the mean changes in AST and ALT concentrations were lower in the pioglitazone group. $^{51}$

According to results of clinical trials, hypoglycemia is less frequent with pioglitazone in combination with metformin than with the combination of sulfonylureas and metformin. These data have been confirmed in shortand long-term studies that have compared pioglitazone in association with metformin with the different sulfonylureas glimepiride ${ }^{52}$ gliclazide,${ }^{53}$ and glibenclamide ${ }^{54}$ administered with metformin.

Data are available that show an association between pioglitazone treatment and moderate reduction of hematocrit and hemoglobin. No evidence exists to show a role of pioglitazone in determining anemia. ${ }^{55}$

A moderate body weight gain is well known in patients treated with TZDs, due to the stimulation of pre-adipocytes differentiation. In clinical studies, treatment with pioglitazone is often associated with moderate increase in body weight in a dose-dependent manner. Body weight increases during the period of treatment with pioglitazone are principally due to an increase in subcutaneous fat. ${ }^{56}$

Combination of pioglitazone with sulfonylureas magnifies the increase in body weight, while combination with metformin seems to moderate this effect. In a 12-month comparator study between pioglitazone and rosiglitazone combined with metformin, no body mass index (BMI) changes were observed in either group. ${ }^{26}$ Combination with pioglitazone showed a small increase in body weight compared with metformin alone, which slightly improved body weight ( $+0.95 \mathrm{~kg}$ vs $-1.36 \mathrm{~kg}$ after 16 weeks). ${ }^{12}$ In other studies, combination treatment of pioglitazone and metformin has been associated with weight gain, comparable to body weight increase obtained with a combination of metformin and gliclazide ( $+1.5 \mathrm{vs} 1.4 \mathrm{~kg}$ when compared to gliclazide after 52 weeks $)^{33}$ and glimepiride (+1.74 vs $1.85 \mathrm{~kg}$, after 28 weeks)..$^{52}$

Finally, a recently published study of the combination of pioglitazone and sibutramine in type 2 diabetes patients intolerant to metformin and treated with sibutramine has shown a substantial equivalence in efficacy and tolerability between the two combination treatments. No changes in BMI and waist circumference were observed in patients treated with pioglitazone plus sibutramine. Results of this study suggest that a combination of pioglitazone with sibutramine might be a useful tool in obese patients with type 2 diabetes intolerant to metformin. ${ }^{57}$

\section{Conclusions and place in therapy}

TZDs represent one of the most promising tools in pharmacotherapy of type 2 diabetes.

As previously reported, in clinical studies pioglitazone has demonstrated efficacy in achieving glycemic control and amelioration of lipid profile. Moreover, improvement in insulin sensitivity is associated with a series of benefits in pro-inflammatory and atherogenetic features in patients with type 2 diabetes mellitus. However, many of the so-called ancillary, antiatherogenetic actions of pioglitazone are still not completely clear and more studies are needed to define the role of pioglitazone in prevention and care of organ damage, endothelial dysfunction, hypertension and cytokines profile. Side effects of treatment with pioglitazone appear moderate and very few subjects have dropped out of clinical trials because of adverse reactions, and adherence to correct prescription of the drug reduces the incidence of intolerance and patient risks. A good clinical evaluation at the beginning of treatment, if monitoring is adequate, enables safer administration of pioglitazone and better results in clinical care. In the past 2 years, published reviews have stimulated debate on the open question of the risk of AMI with TZDs. We consider this issue in this paper as it has raised interesting discussions about pioglitazone safety and efficacy in patients at high $\mathrm{CV}$ risk.

A recent meta-analysis of 42 randomized control trials among 28,443 patients treated with rosiglitazone has shown an increase in the risk of AMI in treated subjects compared with controls (odds ratio 1.43; 95\% CI 1.03-1.98; $\mathrm{p}=0.03$ ). ${ }^{58}$ A subsequent meta-analysis has included randomized clinical 
trials with a specific intention of evaluating CV events in diabetes and non-diabetes subjects (pre-diabetes). Studies included had almost a 12-month follow-up period and provided numerical data of CV side effects. Results of this analysis, according to Nissen et al, have shown that rosiglitazone increases the risk of AMI (relative risk [RR] 1.42\%; CI 95\% 1.06-1.91; $\mathrm{p}=0.02$ ) and congestive HF (RR 2.09; CI 95\% 1.52-2.88; $\mathrm{p}<0.001)$ without raising the risk of $\mathrm{CV}$ mortality (RR 0.90; CI 95\% 0.63-1.26; $\mathrm{p}=0.53$ ). Limitations of collecting data from the studies included in the metaanalysis and the limitations of a meta-analysis itself have been analyzed. ${ }^{59}$ After having considered these data, rosiglitazone label was modified by the FDA in August $2007 .{ }^{60}$

Data from PRO-active support a favorable impact of this molecule on CV risk. In particular, long- term treatment with pioglitazone seems to reduce the risk of AMI. A recent metaanalysis investigated the effect of pioglitazone on CV events. The studies included $(\mathrm{n}=19)$ in this analysis were randomized clinical trials, double-blind, controlled with placebo or active comparator. The primary outcome measure was a composite of death, AMI or stroke, and secondary end-point measures included the incidence of serious HF. Death, AMI or stroke occurred in $4.4 \%$ of patients receiving pioglitazone vs $5.7 \%$ patients receiving control drugs (HR 0.82; CI 95\% $0.72-0.94 ; \mathrm{p}=0.005)$, with a similar degree of reduction in every component of the primary end-point (HR 0.80-0.92), while serious HF was registered in $2.3 \%$ of the patients treated with pioglitazone vs $1.8 \%$ of patients treated with control drug therapy (HR 1.41 CI 95\% 1.14-176; $\mathrm{p}=0.002$ ). In conclusion, pioglitazone appears related to a reduced risk of death, AMI or stroke among a diverse population of patients with type 2 diabetes mellitus. The risk of HF seemed to be higher with pioglitazone, without effects on mortality from HF. ${ }^{61}$ Recently published results from PERISCOPE should encourage physicians to consider pioglitazone as a molecule that substantially improves CV risk factors, even if other studies are required to better establish the pharmacological mechanisms underling these results and to define a precise role of pioglitazone in prevention of $\mathrm{CV}$ events.

Current treatment with pioglitazone in clinical practice has not been excluded by current guidelines, although more care is required in patients at risk of HF or AMI and in women with osteoporosis or at risk of bone fractures. Pioglitazone represents a valid tool in pharmacotherapy in patients characterized by insulin resistance such as obese subjects. No data are available that strongly suggest a superiority of pioglitazone in lipids management in clinical practice; the evidence of a better impact of this molecule on lipids profile should be considered in clinical practice. For a reduction in intima-media thickness, no strong evidence exists for physicians to prefer pioglitazone rather than rosiglitazone. Pioglitazone appears to be well tolerated in association with metformin and sulfonylureas. In particular, in obese patients, the increase in body weight with pioglitazone can be partially reduced in combination with metformin. When pioglitazone is administered to the elderly, a very low incidence of hypoglycemia, the most dangerous side effect in these patients, was observed with monotherapy and when pioglitazone was combined with sulfonylureas, metformin or insulin. ${ }^{62}$ Few data are available about combination with TZDs and DPP-4 inhibitors and/or incretin mimetics. Preliminary conclusions presented in late 2008 at the European Association for the Study of Diabetes in Rome (Italy) suggest a favorable effect of these combinations on glycemic control with a moderate increase in body weight after 52 weeks. ${ }^{63}$ No data are available on the combination of exenatide and pioglitazone. More studies are needed in order to better understand the potential role and safety of these new drugs in association with pioglitazone in the prevention of atherosclerosis.

\section{Patient perspectives}

Pioglitazone is available in tablets $(15,30,45 \mathrm{mg})$ and is usually administered independently of food, one or two times per day. This prescription provides good acceptability for patients with type 2 diabetes mellitus, who are commonly treated with a large number of drugs. As pioglitazone is approved in triple therapy, with metformin and sulfonylureas, many patients can undergo a delay in starting insulin treatment, as pioglitazone therapy is suggested to reduce $\mathrm{HbA}_{1 \mathrm{c}}$ when combination therapy with metformin and sulfonylureas is not able to maintain glycemic control over time. Furthermore, pioglitazone preserves glycemic control over long periods. A common problem with pioglitazone is weight gain, even if it is commonly mitigated by combination with metformin or other therapies and adequate dietary advice.

Pioglitazone falls in the recommended cost-effective range. In a recent analysis, the addition of pioglitazone to existing therapy in high-risk patients with type 2 diabetes was projected to improve life expectancy, quality-adjusted life expectancy and complication rates compared with placebo. ${ }^{64}$

Recently, a fixed dose combination treatment of pioglitazone/metformin/pioglitazone/glimepiride has been approved and introduced on the market. Combination therapy is recommended as first-line treatment in obese 
patients with insulin resistance. The tolerability of a fixed dose seems comparable to the combination of pioglitazone and metformin, and appears well accepted by patients. Moreover, as effects of pioglitazone are usually delayed, fixed dose combination therapy hinders achievement of earlier glycemic control, rather than addition therapy.

\section{Disclosures}

The authors declare no conflicts of interest.

\section{References}

1. Diabetes Atlas, third edition, International Diabetes Federation; 2006. Available online at http://www.eatlas.idf.org/).

2. Ringborg A, Lindgren P, Martinell M, Yin DD, Schon S, Stalhammar J. Prevalence and incidence of type 2 diabetes and its complications 1996-2003-estimates from Swedish population-based study. Diabet Med. 2008;25(10):1178-1786.

3. Grant PJ. Beneficial effects of metformin on haemostasis and vascular function in man. Diabetes Metab. 2003;29(4 Pt 2):S644-S652.

4. Colwell JA. Treatment of the procoagulant sae in type 2 diabetes. Endocrinol Metab Clin North Am. 2001;30(4):1011-1030.

5. UK Prospective Diabetes (UKPDS) Study Group. Effect of intensive blood glucose with metformin on complications in overweight patients with type 2 diabetes (UKPDS 34). Lancet. 1998;352(9131):854-856.

6. Ohkubo Y, Kishikawa H, Araki E, et al. Intensive insulin therapy prevents the progression of diabetic microvascular complications in Japanese patients with non-insulin-dependent diabetes mellitus: a randomized prospective 6-year study. Diabetes Res Clin Pract. 1995;28(2):103-117.

7. ADVANCE Collaborative Group; Patel A, MacMahon S, Chalmers J, et al Intensive blood glucose control and vascular outcomes in patients with type 2 diabetes. $N$ Engl J Med. 2008;358:2560-2572.

8. Available at: www.diabetes.org.

9. Action to control Cardiovascular risk in Diabetes Study Group. Gerstein HC, Miller ME, Byington RP, et al. Effects of intensive glucose lowering in type 2 diabetes mellitus. $N$ Engl $J$ Med. 2008;358:2545-2559.

10. Cugnet-Anceau C, Bauduceau B. Glycaemic control and cardiovascular morbi-mortality: The contribution of the 2008 studies. Ann Endocrinol (Paris). 2009. In press.

11. Sjöholm A, Nyström T. Endothelial inflammation in insulin resistance. Lancet. 2005;365(9459):610-612.

12. Dormandy JA, Charbonnel B, Eckland DJ, et al; PROactive investigators. Secondary prevention of macrovascular events in patients with type 2 diabetes in the PROactive Study (PROspective pioglitAzone Clinical Trial In macroVascular Events): a randomised controlled trial. Lancet. 2005;366:1279-1286.

13. Niessen SE, Nicholls J, Wolski K, et al; PERISCOPE Investigators. Comparison of pioglitazone vs glimepiride on progression of coronary atherosclerosis in patients with type 2 diabetes: the PERISCOPE randomized controlled trial. JAMA. 2008;299(13):1561-1574.

14. Home PD, Pocock SJ, Beck-Nielsen H, et al. Rosiglitazone evaluated for cardiovascular outcome-an interim analysis. $N$ Engl $J$ Med. 2007;357:28-38.

15. Aronoff S, Rosenblatt S, Braithwaite S, Egan JW, Mathisen AL, Schneider RL. Pioglitazone hydrochloride monotherapy improves glycemic control in the treatment of patients with type 2 diabetes: a 6-month randomized placebo-controlled dose-response study. The Pioglitazone 001 Study Group. Diabetes Care. 2000;23(11):1605-1011.

16. Tan MH, Glazer NB, Johns D, Widel M, Gilmore KJ. Pioglitazone as monotherapy or in combination with sulfonylurea or metformin enhances insulin sensitivity (HOMA-S or QUICKI) in patients with type 2 diabetes. Curr Med Res Opin. 2004;20(5):723-728.
17. Scherbaum WA, Göke B; German Pioglitazone Study Group. Metabolic efficacy and safety of once-daily pioglitazone monotherapy in patients with type 2 diabetes: a double-blind, placebo-controlled study. Horm Metab Res. 2002;34(10):589-595.

18. Herz M, Johns D, Reviriego J, et al. A randomized, double-blind, placebo-controlled, clinical trial of the effects of pioglitazone on glycemic control and dyslipidemia in oral antihyperglycemic medication-naive patients with type 2 diabetes mellitus. Clin Ther. 2003;25(4):1074-1095.

19. Rosenblatt S, Miskin B, Glazer NB, Prince MJ, Robertson KE; Pioglitazone 026 Study Group. The impact of pioglitazone on glycemic control and atherogenic dyslipidemia in patients with type 2 diabetes mellitus. Coron Artery Dis. 2001;12(5):413-423.

20. Pavo I, Jermendy G, Varkonyi T T, et al. Effect of pioglitazone compared with metformin on glycemic control and indicators of insulin sensitivity in recently diagnosed patients with type 2 diabetes. $J$ Clin Endocrinol Metab. 2003;88(4):1637-1645.

21. Schernthaner G, Matthews DR, Charbonnel B, Hanefeld M, Brunetti P; Quartet Study Group. Efficacy and safety of pioglitazone versus metformin in patients with type 2 diabetes mellitus: a double-blind, randomized trial. J Clin Endocrinol Metab. 2004;89(12):6068-6076.

22. Walter H, Lübben G. Potential role of oral thiazolidinedione therapy in preserving beta-cell function in type 2 diabetes mellitus. Drugs. 2005;65(1):1-13.

23. Tan MH, Johns D, Strand J, Halse J, et al. GLAC Study Group. Sustained effects of pioglitazone vs. glibenclamide on insulin sensitivity, glycae mic control, and lipid profiles in patients with Type 2 diabetes. Diabet Med. 2004;21(8):859-866.

24. Tan M, Johns D, González Gálvez, et al; GLAD Study Group. Effects of pioglitazone and glimepiride on glycemic control and insulin sensitivity in Mexican patients with type 2 diabetes mellitus: A multicenter, randomized, double-blind, parallel-group trial. Clin Ther. 2004;26(5):680-693.

25. Rizzo M, Christ ER, Rini GB, Spinas GA, Berneis K. The differential effects of thiazolidindiones on atherogenic dyslipidemia in type 2 diabetes: what is the clinical significance? Expert Opin Pharmacother. 2008;9(13):2295-2303.

26. Derosa G, D'Angelo A, Ragonesi PD, et al. Metabolic effects of pioglitazone and rosiglitazone in patients with diabetes and metabolic syndrome treated with metformin. Intern Med J. 2007;37(2):79-86.

27. Derosa G, Cicero AF, Gaddi A, et al. Metabolic effects of pioglitazone and rosiglitazone in patients with diabetes and metabolic syndrome treated with glimepiride: a twelve-month, multicenter, double-blind, randomized, controlled, parallel-group trial. Clin Ther. 2004;26(5):744-754.

28. Yki-Järvinen H. Thiazolidinediones. N Engl J Med. 2004;351(11): 1106-1118.

29. Negro R, Dazzi D, Hassan H, Pezzarossa A. Pioglitazone reduces blood pressure in non-dipping diabetic patients. Minerva Endocrinol. 2004;29(1):11-17.

30. Derosa G, Cicero AF, D'Angelo A, et al. Thiazolidinedione effects on blood pressure in diabetic patients with metabolic syndrome treated with glimepiride. Hypertens Res. 2005;28(11):917-924.

31. Derosa G, Fogari E, Cicero AF, et al. Blood pressure control and inflammatory markers in type 2 diabetic patients treated with pioglitazone or rosiglitazone and metformin. Hypertens Res. 2007;30(5): 387-394.

32. Hanefeld M, Brunetti P, Schernthaner GH, Matthews DR, Charbonnel BH; QUARTET Study Group. One-year glycemic control with a sulfonylurea plus pioglitazone versus a sulfonylurea plus metformin in patients with type 2 diabetes. Diabetes Care. 2004;27(1): 141-147.

33. Matthews DR, Charbonnel BH, Hanefeld M, Brunetti P, Schernthaner G. Long-term therapy with addition of pioglitazone to metformin compared with the addition of gliclazide to metformin in patients with type 2 diabetes: a randomized, comparative study. Diabetes Metab Res Rev. 2005;21(2):167-174. 
34. Forst $\mathrm{T}$, Hohberg C, Fuellert SD, et al. Pharmacological PPARgamma stimulation in contrast to beta cell stimulation results in an improvement in adiponectin and proinsulin intact levels and reduces intima media thickness in patients with type 2 diabetes. Horm Metab Res. 2005;37(8):521-527.

35. Mazzone T, Meyer PM, Feinstein SB, et al. Effect of pioglitazone compared with glimepiride on carotid intima-media thickness in type 2 diabetes: a randomized trial. JAMA. 2006;296(21):2572-2581.

36. Horio T, Suzuki M, Takamisawa I, et al. Pioglitazone-induced insulin sensitization improves vascular endothelial function in nondiabetic patients with essential hypertension. Am J Hypertens. 2005;18(12 Pt 1):1626-1630.

37. Nakayama T, Komiyama N, Yokoyama M, et al. Pioglitazone induces regression of coronary atherosclerotic plaques in patients with type 2 diabetes mellitus or impaired glucose tolerance: A randomized prospective study using intravascular ultrasound. Int J Cardiol. 2008 Sep 23. [Epub ahead of print].

38. Derosa G, D'Angelo A, Ragonesi PD, et al. Metformin-pioglitazone and metformin-rosiglitazone effects on non-conventional cardiovascular risk factors plasma level in type 2 diabetic patients with metabolic syndrome. J Clin Pharm Ther. 2006;31(4):375-383.

39. Einhorn D, Rendell M, Rosenzweig J, et al. Pioglitazone hydrochloride in combination with metformin in the treatment of type 2 diabetes mellitus: a randomized, placebo-controlled study. The Pioglitazone 027 Study Group. Clin Ther. 2000;22(12):1395-1409.

40. Nesto RW, Bell D, Bonow RO, Fonseca V, et al. Thiazolidinedione use, fluid retention and congestive heart failure. A consensus statement from the American Heart association and American Diabetes Association. Diabetes Care. 2005;27(1):256-263.

41. Emoto M, Anno T, Sato Y, et al. Troglitazone treatement increases plasma vascular endothelial growth factor in diabetic patients and its mRNA in 3T3-L1 adipocytes. Diabetes. 2001;50:1166-1170.

42. El Muayed M, Lavis VR, Safi HJ, et al. Use of glitazone in cardiac patients: a case for B-type natriuretic peptide monitoring? Am J Cardiol. 2004;93:600-602.

43. Sambanis C, Tziomalos K, Kountana E, et al. Effect of pioglitazone on heart function and $\mathrm{N}$-terminal pro-brain natriuretic peptide levels of patients with type 2 diabetes. Acta Diabetol. 2008;45(1):23-30.

44. Erdmann E. Heart failure with thiazolidinedione treatment: what do we know today? Dtsch Med Wochenschr. 2009;134(4):154-158.

45. McDonough AK, Rosenthal RS, Cao X, Saag KG.The effect of thiazolidinediones on BMD and osteoporosis. Nat Clin Pract Endocrinol Metab. 2008;4(9):507-513.

46. Schwartz AV, Sellmeyer DE, Vittinghoff E, et al. Thiazolidinedione use and bone loss in older diabetic adults. $J$ Clin Endocrinol Metab. 2006;91:3349-3354.

47. Watanabe S, Takeuchi Y, Fukumoto S, Fujita H, et al. Decrease in serum leptin by troglitazone is associated with preventing bone loss in type 2 diabetic patients. J Bone Miner Metab. 2003;21:166-171.

48. Glintborg D, Andersen M, Hagen C, et al. Association of pioglitazone treatment with decrease bone mineral density in obese premenopausal patients with policistic ovary syndrome: a randomized, placebo controlled trial. J Clin Endocrinol Metab. 2008;93(5):1696-1701.
49. Kempna P, Hofer G, Mullis PE, Fluck CE. Pioglitazone inhibits androgen production in NCI-H295R cells by regulating gene expression of CYP17 and HSD3B2. Mol Pharmacol. 2007;71:787-798.

50. Gholamine B, Shafiei M, Motevallian M, Mahmoudian M. Effects of pioglitazone on erectile dysfunction in sildenafil poor-responders: a randomized, controlled study. J Pharm Pharmaceut Sci. 2008; 11(1):22-31.

51. Spanheimer R, Perez A, Kupfer S, et al. The effects of pioglitazone vs glyburide on glycemic control in patients with type 2 diabetes in a 3-year randomized double-blind trial. Diab Vasc Dis Res. 2006;3(1):39-44.

52. Umpierrez G, Issa M, Vlajnic A. Glimepiride versus pioglitazone combination therapy in subjects with type diabetes inadequately controlled on metformin monotherapy: results of a randomized clinical trial. Curr Med Res Opin. 2006;22:751-759.

53. Charbonell BH, Schernthaner G, Brunetti P, et al. Long-term efficacy and tolerability of add-on pioglitazone therapy to failing monotherapy compared with addition of gliclazide or metfomin in patients with type 2 diabetes. Diabetologia. 2005;48:1093-1104.

54. Kupfer S, Spanheimer R, Perez A, et al. Cardiovascular safety profile of pioglitazone vs glyburide: results from a 3-year, randomized, double blind trial [abstract]. 66th Annual Scientific Session of The American Diabetes Association.

55. Hanefeld M, Belcher G. Safety profile of pioglitazone. Int J Clin Pract Suppl. 2001;121:27-31.

56. Smith SR, De Jonge L, Volaufova J, Li Y, Xie H, Bray GA. Effect of pioglitazone on body composition and energy expenditure: a randomized controlled trial. Metabolism. 2005;54(1):24-32.

57. Derosa G, Mereu R, Salvadeo SAT, et al. Pioglitazone metabolic effect in metformin-intolerant obese patients treated with sibutramine. Inter Med. 2009;48:265-271.

58. Nissen SE, Wolsk1 K. Effect of rosiglitazone on the risk of myocardial infarction and death from cardiovascular causes. $N$ Engl $J$ Med. 2007;356:2457-2471.

59. Singh S, Loke YK, Furberg CD. Long term risk of cardiovascular events with rosiglitazone. A meta-analysis. JAMA. 2007;298 (10):1189-1195.

60. Division of Metabolism and Endocrine Products and Office of Surveillance and Epidemology, US Food and Drug Administration FDA Briefing Document: joint meeting of the Endocrinologic and and Metabolic Drugs Advisory Committee; available at www.fda.gov.

61. Lincoff AM, Wolski K, Nicholls SJ, Nissen SE. Pioglitazone and the risk of cardiovascular events in patients with type 2 diabetes mellitus. A meta-analysis of randomized trials. JAMA. 2007;298 (10):1180-1187.

62. Rajagopalan P, Perez A, Ye Z, Khan M, Murray FT. Pioglitazone is effective therapy for elderly patients with type 2 diabetes mellitus. Drugs Aging. 2004;21:259-271.

63. Maegawa H, et al. Sitagliptin added to ongoing treatment with pioglitazone study up to 52 weeks in Japanese patients with T2DM [abstract] European Association for the Study Diabetes. 2008; Abstract 910.

64. Valentine WJ, Tucker D, Palmer AJ, Minshall ME, Foos V, Silberman C. Long-term cost-effectiveness of pioglitazone versus placebo in addition to existing diabetes treatment: A US Analysis Based on PROactive Value Health. 2008 Jul 24. [Epub ahead of print].

Diabetes, Metabolic Syndrome and Obesity: Targets and Therapy

Dovepress

\section{Publish your work in this journal}

Diabetes, Metabolic Syndrome and Obesity: Targets and Therapy is an international, peer-reviewed open-access journal committed to the rapid publication of the latest laboratory and clinical findings in the fields of diabetes, metabolic syndrome and obesity research. Original research, review, case reports, hypothesis formation, expert opinion and commentaries are all considered for publication. The manuscript management system is completely online and includes a very quick and fair peer-review system, which is all easy to use. Visit http://www.dovepress.com/testimonials.php to read real quotes from published authors. 